\title{
European Society of Gastrointestinal Endoscopy (ESGE) guideline: the use of electrosurgical units
}

Authors

Institutions
J. F. Rey' ${ }^{1}$, U. Beilenhoff ${ }^{2}$, C. S. Neumann ${ }^{3}$, J. M. Dumonceau ${ }^{4}$

Institut Arnault Tzanck, St. Laurent du Var, France

European Society of Gastroenterology and Endoscopy Nurses and Associates (President), Ulm, Germany

European Society of Gastroenterology and Endoscopy Nurses and Associates (Past President), Birmingham, United Kingdom

${ }^{4}$ Division of Gastroenterology and Hepatology, University Hospital of Geneva, Geneva, Switzerland
Bibliography

Dol http://dx.doi.org/

10.1055/s-0030-1255594

Published online

15 July 2010

Endoscopy 2010; 42:

764-771 @ Georg Thieme

Verlag KG Stuttgart · New York

ISSN 0013-726X

Corresponding author

J. M. Dumonceau, MD, PhD

Division of Gastroenterology and Hepatology

Geneva University Hospitals

Micheli-du-Crest Street 24

1205 Geneva

Switzerland

Fax: +41-22-3729366

jmdumonceau@hotmail.com
Electrosurgery is used in the majority of endoscopic therapeutic procedures. An understanding of the fundamental electrosurgical principles and various settings available on electrosurgical units is essential for the safe and effective use of electrosurgery during endoscopy. The aims of these technical guidelines are to: (1) expose physical principles relevant to the understanding of electrosurgery during endoscopy; (2) describe and

\section{Introduction}

\section{$\nabla$}

Electrosurgery in endoscopy is an old technique, with urologists already using it in an aqueous environment in 1926 [1]. Despite the availability of electrosurgical units (ESUs) with better safety profiles, some practitioners still use electrosurgery under conditions that might be hazardous with old technologies. In particular, ESUs with spark gap technology pre-date the solid-state units introduced in 1968, and are no longer acceptable. Also obsolete are "grounded" or "ground referenced" electrosurgical systems where the ground, not the generator, completes the circuit. The replacement of this technology with isolated, balanced, and return monitoring systems has virtually eliminated any risk to patients that may arise from current division, in which all or part of the current seeks ground through paths other than the neutral electrode, which could result in alternate site burns. In addition to markedly greater patient safety, newer ESUs provide opportunities for enhanced bipolar outputs and sophisticated neutral electrode safety monitoring [2]. Moreover, the development of microprocessor-controlled ESUs has enabled more constant, reliable or predictable qualities of current waveform, power output, and tissue effects. provide practical recommendations regarding electrosurgical units that are commonly in use; (3) discuss the clinical relevance of technologies recently implemented in newer electrosurgical units; and (4) review factors relevant to commonly performed therapeutic procedures, including polypectomy, sphincterotomy, contact thermal hemostasis, and argon plasma coagulation.

\section{Technical background \\ $\nabla$}

\subsection{Electricity and tissue effects}

Electrosurgery is based on the transformation of energy from high frequency electric current into heat, with the resulting effect of cutting and/or coagulating tissue at the point of current application [3]. As current passes through tissue, electrons collide with various tissue components. During these collisions, a certain amount of energy is dissipated depending on the nature of the material traversed, which results in a rise in temperature. Electrosurgical waveforms may be set to promote two types of tissue effects, namely coagulation (temperature rises within cells, which then dehydrate and shrink) or cut (heating of cellular water occurs so rapidly that cells burst). The proportion of cells coagulated to those cut can be varied, resulting in a "blended" or "mixed" effect. The rise in temperature is governed by Joule's law:

$\mathrm{Q}=\mathrm{I}^{2} \times \mathrm{R} \times \mathrm{t}$

where $Q$ is the heat generated by a current of constant intensity (I) flowing through a conductor of electrical resistance $(\mathrm{R})$, for a time $(\mathrm{t})$ [3].

Tissue electrical resistance mostly depends on the degree of vascularization and water content. For example, bone and fat present a higher electrical resistance than skin and muscles. When electrosurgery is applied, progressive tissue desiccation increases tissue resistance, which reduces current intensity. 


\section{Key points and recommendations}

\section{a) Protection measures for the patient}

The electrosurgical unit (ESU) should only be used by medical personnel after appropriate training.

- Inspect the ESU for damage, including insulation of all cables and electrodes, missing components, and working lights and sounds (set on an audible level) prior to use.

- The ESU should stand firm; no fluids should be placed on top of the ESU.

- Do not use worn out or defective active electrodes, forceps or scissors.

- Do not repair active electrodes, forceps or scissors.

- Do not use the ESU in the presence of flammable material or substances (e.g. alcohol or nitrous oxide).

- The patient must be insulated against all electrically conductive parts. Make sure that the patient does not come into contact with other metal parts not insulated from the ground (e.g. operating table), although this concern is greatly diminished by the use of isolated and/or balanced ESUs.

- Place the patient on a dry, electrically insulating layer.

- Pacemaker or defibrillator (all types): seek advice from competent authority prior to endoscopy. Permanent ECG monitoring is recommended in these patients during electrosurgery. The use of bipolar applications might minimize possible complications. If a monopolar electrosurgical system is used, position the neutral electrode as close as possible to the active electrode. Direct contact with the implanted device and the leads should be avoided.

- The power settings should be adapted to the type of procedure, tissue structure, patient body mass index, endotherapy instrument used, and manufacturers' recommendations. Always use the lowest power setting possible that will accomplish the desired tissue effect.

- Before activating the ESU, the power settings should be rechecked and verbally confirmed between the endoscopist and the assistant.

- If current is not required, keep the foot away from the pedal to prevent accidental pressing, or disconnect the electrode from the ESU.

- If inadequate current output is observed, stop the procedure immediately. Use the power switch as emergency stop for malfunctions. Leakage of current may be the result of a malfunctioning endotherapy instrument, ESU or broken insulation of the endoscope and may cause user and/or patient burns. Electrosurgical units that constantly monitor leakage of current may help to ensure user and patient safety. b) Staff safety

- Avoid contact with the neutral electrode.

- When applying current, always be sure to wear gloves and touch the equipment or patient body with the entire palm of the hand, not with a single finger.

- Electrosurgical equipment needs to be grounded in order to minimize interference with videoendoscopic systems.

- Smoke generated during electrosurgical procedures can be irritating and potentially harmful to personnel; surgical masks and adequate ventilation of smoke may be useful.

c) Neutral electrode

- Only patient neutral electrodes (plates or grounding pads) recommended by the ESU manufacturer should be used. For example, some ESUs require split type plates to monitor the quality of contact between the plate and the patient; singleuse plates should not be reused.

- Check expiration date (if expired patient plates are used, the adhesive may fail to maintain contact with the patient's skin and burns may result).

- Check the patient plate for any damage/modification or sharp edges.

- The neutral electrode should not be attached over some structures, including bony protuberances, metal implants or prosthesis, skin folds, scar tissue, hairy areas, any form of skin discoloration/injury, limbs with a restricted blood supply, adjacent to ECG electrodes or onto pressure areas/points.

- The neutral electrode should be attached over well perfused muscle tissue; the skin must be clean, dry, and free of hair to avoid loss of contact between the plate and the skin. The electrode should not be completely wrapped around a limb. Overlapping needs to be avoided. Ensure that the neutral electrode has full patient-skin contact.

- The patient plate should be of appropriate size for the patient weight and should never be cut to size.

- Patient plates that have once been removed from the patient skin have to be replaced by new ones.

d) Special situation: polypectomy or EMR

- Adjust settings according to particular conditions (e.g. low power settings for small bowel and cecum).

- If polypectomy snare sticks in a polyp, increase cutting (see text above).

- Do not touch metal parts, such as clips, with snare when applying current.

- Do not touch the scope with metal parts of endotherapy instruments.

- Watch out that snare tip does not accidentally touch the bowel wall opposite to mucosectomy.

- Avoid deep coagulation of muscle layer (risk of late perforation).

- Before applying current, make sure that the muscularis propria is not entrapped in the snare loop. 


\subsection{Electrical circuit}

Depending on the clinical indication and type of device used, energy may be transmitted by ESUs in monopolar or bipolar mode.

- In monopolar mode, the electrical current passes from the active electrode to the target tissue, through the patient's body, to finally exit the patient through a large dispersive neutral electrode (also called a patient plate or return electrode)

\section{(๑ Fig. 1).}

- In bipolar mode, the endoscopic device contains both the active and the neutral electrodes in close proximity to each other. The electrical current passes directly from one electrode to the other through a small amount of tissue in contact with both electrodes. The current does not pass through the rest of the patient's body and no patient plate is required ( $\bullet$ Fig. 2 ).

\subsection{Current density and rise in temperature}

Current density is the amount of electrical current per unit area of cross section. In a monopolar circuit, current density is relatively high at the point of tissue contact with the active electrode due to the small contact area; current density is considerably lower at the point of contact between the patient and the neutral electrode because the larger surface area of the latter disperses the current returning to the ESU.

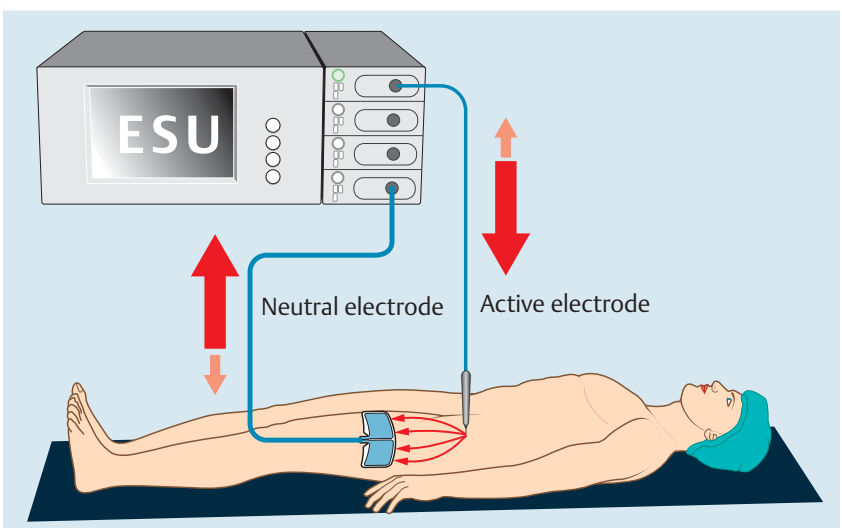

Fig. 1 Monopolar electrosurgery. Electricity flows from the active electrode (usually inserted through the working channel of the endoscope) to the neutral electrode placed on patient skin.

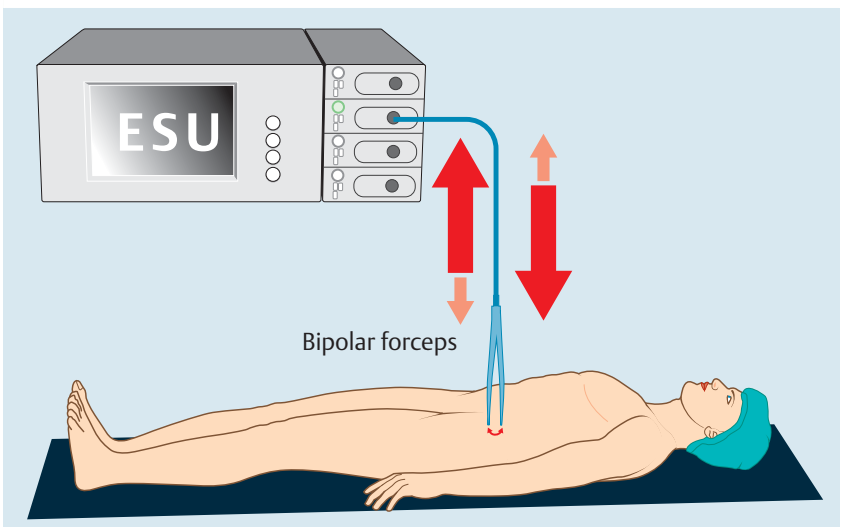

Fig. 2 Bipolar electrosurgery. Electricity flows from the active to the neutral electrode, both of them being located in close proximity on a single endoscopic device.
If we assume that tissue electrical resistance is uniform and heat conduction is negligible, the increase in tissue temperature is directly related to the amount of electrical energy absorbed by the tissue. This amount $(\mathrm{QE})$ can be expressed by the equation:

$\mathrm{QE}=\left(\mathrm{I}^{2} \times \mathrm{R}\right) / \mathrm{S}$

where $\mathrm{R}$ represents tissue electrical resistance, I the current intensity, and $S$ the surface of tissue in contact with the electrode. When electrical current is applied for 1 second, tissue temperature increases according to the following equation (assuming that all the electrical energy is transformed into heat):

$\Delta \mathrm{T}=(\mathrm{K} \times \mathrm{QE}) / \mathrm{S}=\left(\mathrm{I}^{2} \times \mathrm{K} \times \mathrm{R}\right) / \mathrm{S}^{2}$

where $\mathrm{K}$ is a tissue-specific heat parameter.

Tissue temperature increases with the square of the current intensity. QE may be adjusted to cause an increase in tissue temperature at the level of contact with the active electrode while thermal effect at the level of the neutral electrode, which is considerably larger, is negligible. Conversely, if the patient is in contact with a small portion of the neutral electrode, or if the neutral electrode is not correctly oriented, the temperature at this level may rise and cause skin burn ( $\bullet$ Fig. 3 ).

Therefore, it is important to ensure that the patient skin is in good contact with the entire surface of the neutral electrode and avoid any interfering matter (e.g. cream, hair, scar) that could decrease conductivity and cause skin burns. A "contact quality monitor" (CQM) ESU system, combined with split patient plates, helps to prevent the development of such burns as the CQM shuts down power delivery if the contact surface becomes too small. The neutral electrode should also be positioned as close as possible to the active electrode to reduce passage of electrical current through the patient's body.

\subsection{Current frequency}

Human myocardium is sensitive to household alternating electrical currents of low frequency, with a risk of ventricular fibrillation and cardiac arrest. High frequency current $(>300 \mathrm{kHz})$ is used in electrosurgery because myocardium sensitivity decreases with increasing current frequencies. However, the risk of electrostatic losses increases with increasing frequencies, thus reducing the efficiency of current application and increasing the risk of burns to either the operator or the patient. Therefore, high frequency currents in the range of $300-1000 \mathrm{kHz}$ are usually employed during electrosurgery.

\subsection{Current waveforms}

High frequency currents generated by ESUs consist of one of the two following types.

- Pure sinusoidal current waveform ( $\bullet$ Fig. 4 a):

- the "crest factor" (ratio of the peak to the root-mean-square voltage) is constant at 1.4 for every sinus waveform;

- higher peak voltages provide more intense coagulation/ hemostasis effects;

- if the voltage peak $(\mathrm{Vp})$ is below $200 \mathrm{~V}$, it is insufficient for a cutting effect, but it is ideal for soft coagulation;

- Vp of approximately $300 \mathrm{~V}$ enable a "pure" cutting effect with the smallest possible coagulation effect.

- Amplitude modulated current waveforms ( $\bullet$ Fig. 4b):

- the crest factor varies between 1.5 and 8 , with increasing crest factors providing deeper coagulation effect;

- in some ESUs, cutting and coagulation currents with these characteristics are named "Blend Cut" or "Dry Cut" and "Fulgurate," "Forced Coag” or "Spray Coag," respectively. 


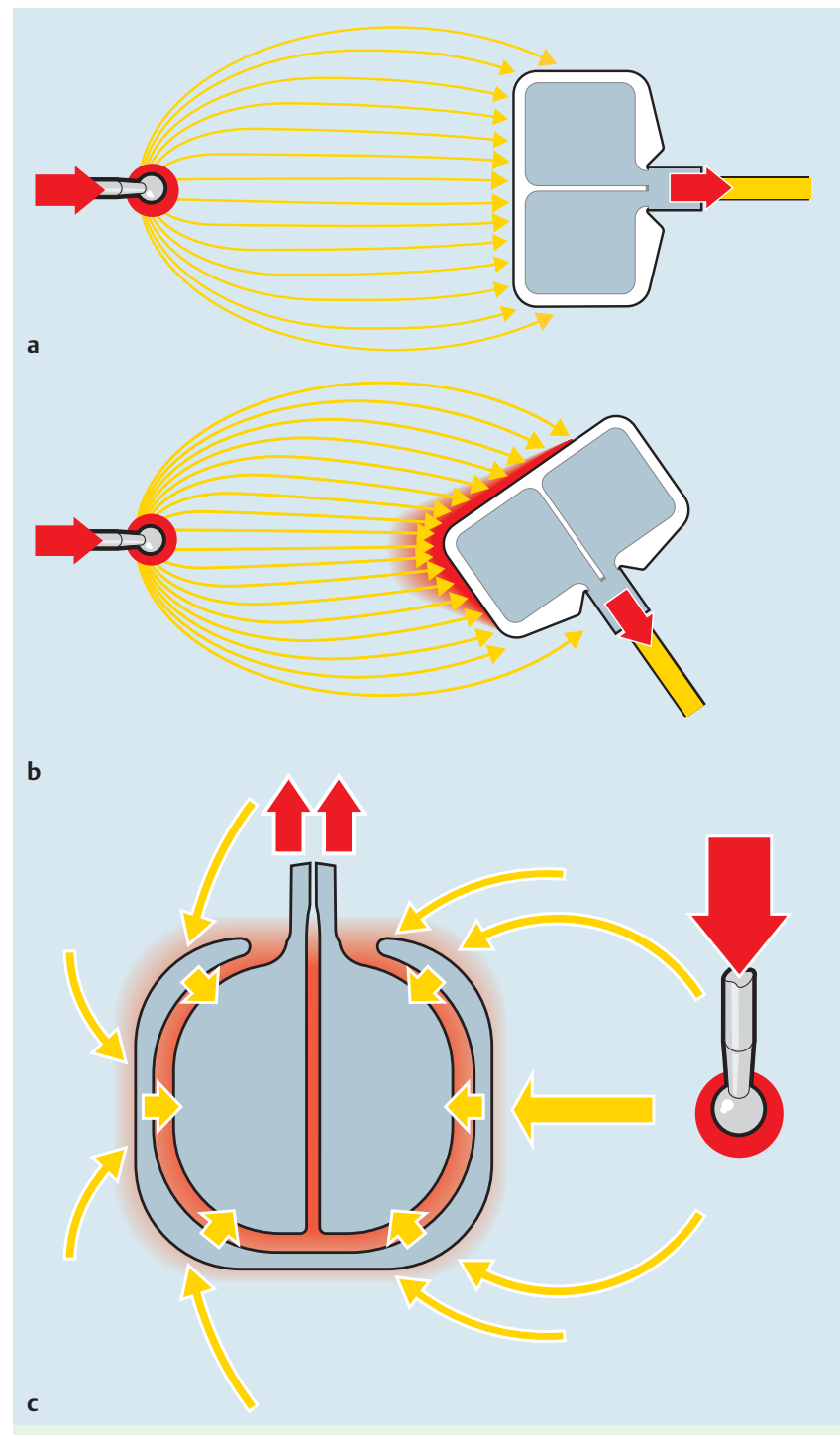

Fig. 3 Placement of the neutral electrode ("patient plate") for monopolar electrosurgery. a Correct plate positioning, with its "long edge" directed towards the operative field. b Wrong plate positioning, with the "short edge" directed towards the operative field (high current density at the plate corner might cause extended heating or "asymmetry alarm"). c Neutral electrode equipped with an equipotential ring requiring no particular plate orientation.

Some ESUs combine cutting and coagulation waveforms in one mode that alternates each type of waveform (e.g. Endocut, Pulsecut) $($ Fig. 5).

\section{Electrosurgical units}

\section{$\nabla$}

All ESUs available for endoscopic use in Europe provide basic features including current waveforms for cutting and coagulation of several characteristics, blended currents, as well as CQM (provided that an adequate neutral electrode is used). In addition to this, some ESUs provide modes that alternate cutting and coagulation phases (and, in some units, adaptation of the current according to continuous measurements made during the procedure, making a closed-loop feedback) or a bipolar cutting mode. Some ESUs allow the user to modify many parameters of the current selected

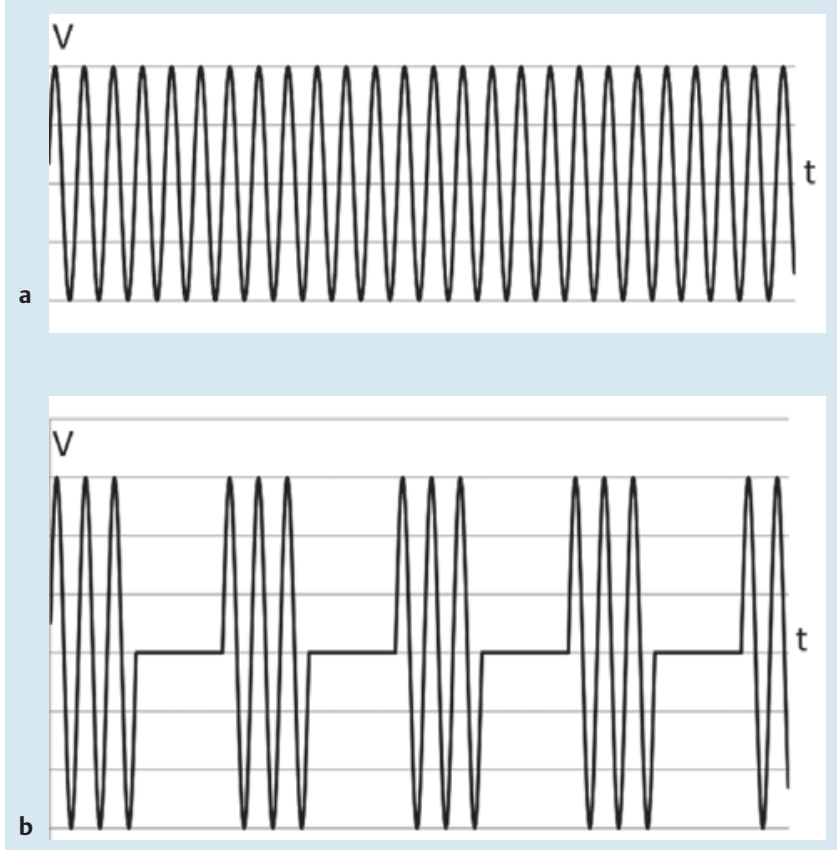

Fig. 4 Current waveforms used in electrosurgery. a Pure sinus current waveform: continuous sinusoidal waveform with a crest factor (ratio of the peak to the root-mean-square voltage) of 1.4; $\mathbf{b}$ Modulated current waveform: crest factor may vary between 1.5 and 8 .

( Fig. 6), to connect optional modules that provide argon plasma coagulation (APC) or irrigation through ancillary devices.

Some ESUs also support the use of a single device that allows both injection and dissection during endoscopic submucosal dissection (ESD). The size of the ESU may also be an important factor in small endoscopy rooms. Additional information on ESUs is available from Morris et al. [4].

\section{Interference with other electrical equipment \\ $\nabla$}

Modern endoscopy units are equipped with several electrical devices and appliances that may potentially give rise to electrical interferences. For example, the electrocardiograph (ECG) can create a false ground and skin burns can develop at points of contact with the electrodes if the electrode cables are placed close to, or entwined with, cables leading to the active electrode.

In a patient with a cardiac pacemaker or an implantable cardioverter-defibrillator, constant supervision must be maintained when applying high frequency current, particularly in monopolar mode, as it can result in cardiac arrest in a truly pacemaker-dependent patient. Monitoring of the patient ECG must include the possibility to detect pacemaker discharges (artifact filter should be disabled) and peripheral pulse should be monitored using a pulse oximeter [5]. Defibrillator equipment should be readily accessible. A preprocedure electrocardiogram should be obtained and, if necessary, the device should be reset in ventricular asynchronous (VOO) mode. This type of adjustment should be made by the appropriate personnel (e.g. cardiologist or pacemaker technician), and once the endoscopic procedure is terminated, the device is interrogated and reset to the preprocedure mode. During the procedure, bipolar electrosurgery is preferred, if feasible. If the monopolar mode is used, pure cut current may be preferable and the neutral electrode is positioned in a location 

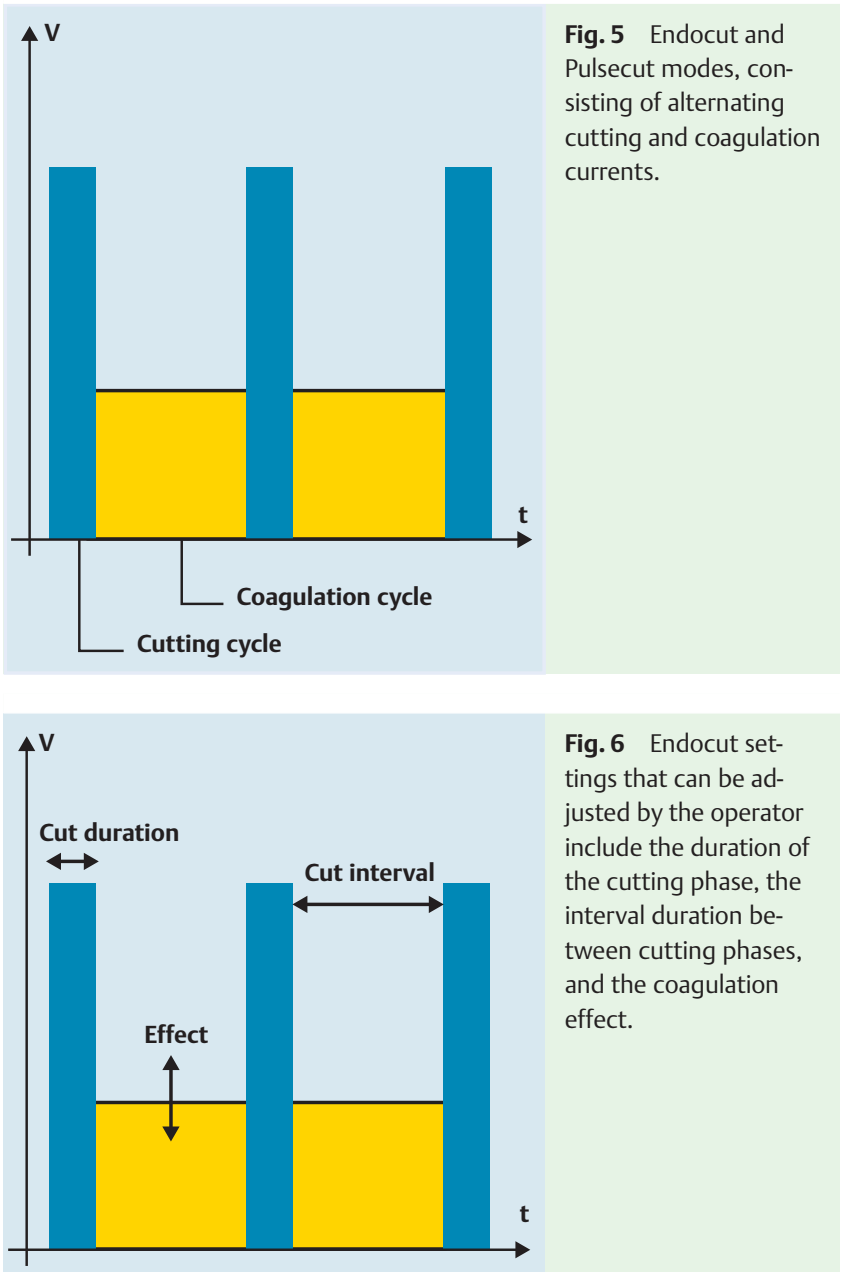

that minimizes the current crossing the pacemaker-heart circuit. In emergent situations, placing a magnet close to the device temporarily "reprograms" the pacer into asynchronous mode if the device has a magnet mode (most have). A magnet marketed by one pacemaker manufacturer is usually effective with other brands of pacemaker.

\section{Electrosurgical settings}

$\nabla$

Heat generated in the digestive mucosa is directly proportional to the square of current intensity (e.g. if current intensity is doubled, the heat generated increases by a factor of 4). Understanding this relationship between current intensity, density, and heat is essential for adequate ESU operation. As an example, the thermal effect of current application to the base of a polyp is shown in - Fig. 7.

All other things being equal, the larger the base of the polyp or the snare wire, the more energy will be required to section it. Manufacturer-recommended settings for various applications are presented in the Appendices available on line. Settings can be adjusted before and during the procedure according to the endoscopist preferences and desirable therapeutic response. For example, in the Endocut mode, no coagulation is applied between cutting cycles if the lowest "effect" level is selected, which may be useful at limiting coagulation-related damage in the cecum. During ESD using a Flush knife, coagulation may be switched from "Soft Coag" to "Forced Coag" to treat a bleeding vessel.

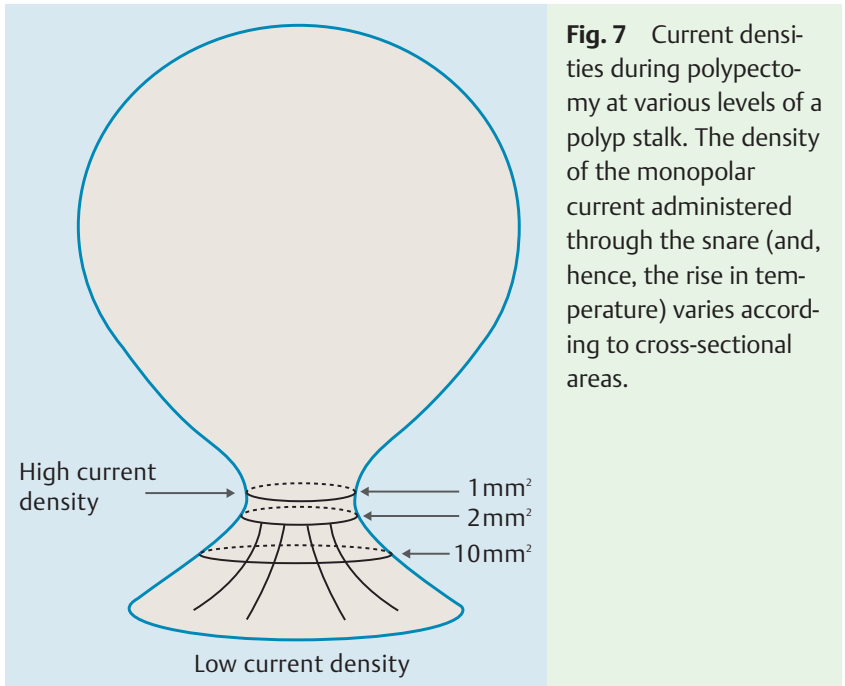

\section{Practical applications \\ $\nabla$}

\subsection{Polypectomy}

Although this section deals with colon polypectomy, the principles described here are applicable to other polypectomy sites. Bleeding is the most frequent complication of polypectomy, occurring in $1 \%-4 \%$ of cases, compared with a $0.5 \%$ risk for perforation [6].

\subsubsection{Polypectomy snares}

Polypectomy snares of different size, shape, and wire characteristics are commercially available. A snare with a particular diameter and shape is selected according to the size of the polyp and bowel features (e.g. a small oval snare is preferable for removing a small polyp in a diverticular-laden, narrow, colonic segment). It is important to have a snare design that facilitates capture of the lesion; rotatable snares may facilitate polyp grasping. Features of the snare wire may also influence the electrosurgical outcome, as a thin or monofilament wire will favor cutting whereas a thick or braided wire will favor coagulation. As manufacturer-recommended settings are not uniformly based on a particular type of snare and wire characteristic, ESU settings may need to be adjusted accordingly, depending on the type of snare used (e.g. by testing on a piece of meat ex vivo). Thicker snares require higher power settings (powerful cut with limited coagulation). Any alteration to the snare wire (especially fraying) may lead to an increase in the contact area between the polyp and the snare, resulting in decreased efficiency. Also, the speed of snare closure directly affects the efficacy of the current applied and outcome. For example, closing the snare too fast might result in insufficient tissue coagulation, resulting in bleeding.

\subsubsection{Current application}

Prototype bipolar snares have been assessed in experimental studies, but monopolar snare polypectomy is currently the standard of practice [7]. The use of blended or coagulation current, rather than pure cut waveforms, is recommended for polypectomy. In a large multicenter study encompassing more than 9000 polypectomies, the use of pure cut current was associated with the highest odds ratio for immediate postpolypectomy bleeding along with inadvertent cold polypectomy (odds ratio of 6.95 and 71.15, respectively) [8]. In a single-center report of a large series of polypectomies using pure cut current, the bleeding 
rate was acceptable (3.1\% of patients) but the authors insisted on the need to have hemoclips readily available as these were used in $12 \%$ of their patients [6].

In a retrospective study that compared blended with coagulation current, the incidence of postpolypectomy bleeding was similar in both groups of patients but the time interval to bleeding was different. Immediate postpolypectomy bleeding was more frequent with blended current, whereas delayed bleeding (2-8 days postpolypectomy) occurred more frequently with coagulation current [9]. Patient circumstances may influence the selection of a particular current waveform, as it may be more practical to treat immediate rather than delayed bleeding.

The type of current used may also affect the adequacy of resected polyps for histopathological examination. A retrospective study compared polypectomy specimens obtained using two different current settings: "blended 2" current at a setting of $30 \mathrm{~W}$ (model not specified; ValleyLab, Boulder, Colorado, USA) and Endocut with cutting and coagulation settings of $120 \mathrm{~W}$ (ICC 200, Erbe, Tübingen, Germany). A dedicated pathologist blinded to the resection technique found that resection margins were evaluable in $60.3 \%$ vs. $75.7 \%$ of the polyps resected using blended 2 vs. Endocut current, respectively $(P=0.046)$ [10].

In the absence of firm evidence-based data, no specific recommendation can be made regarding the selection of a particular electrosurgical current for polypectomy except for the avoidance of pure cut current. A recent survey found that identical proportions of endoscopists used coagulation current (46\%) and blended current (46\%) for polypectomy, whereas only a small number of responders used pure cut $(3 \%)$ or modified the current during polypectomy (4\%) [11].

Practical recommendations regarding the technique of snare polypectomy include the following.

- Once seized with a snare, the polyp should be tented away from the colon wall in order to decrease the risk of perforation.

- During polypectomy, tissue resistance will increase as desiccation occurs, and sectioning the central part of the polyp base is therefore slower.

- Contact between the surface of the polyp and the bowel wall should be avoided to minimize contralateral burn. This may be difficult with a very large polyp, but wiggling the polyp during current application will minimize damage of normal tissue in contact with the polyp being resected.

- A risk of snare entrapment in desiccated tissue exists for very large polyps. If the area of contact between the snare and the tissue is large, current density may be too low to efficiently section the polyp base. The natural tendency is to increase snare tightening around the polyp but this may section the tissue without providing enough coagulation or increase the area of contact between the snare and the tissue surrounding the metal wire, further decreasing cutting efficiency. This situation can best be avoided by not overly tightening the snare (strangulation) or attempting polypectomy on a large pedicle with too low a power setting. Some theorize that using a cut mode that provides automatic power adjustment (e.g. Endocut) may be of benefit, but this has not been studied in a randomized trial. Otherwise, if entrapment occurs, it may be beneficial to pause to allow the tissue to reabsorb some water and then to adjust the power settings of the ESU in a stepwise manner.

\subsubsection{Resection of small polyps with a hot biopsy forceps}

Polypectomy using a hot biopsy forceps should be avoided as this technique leaves residual neoplastic tissue in situ in $15 \%$ of cases and the pathological examination of resected specimens is severely hampered by thermal artifacts [12]. If the technique is nevertheless used to sample a large polyp or to resect a polyp of less than $5 \mathrm{~mm}$, the forceps should be tented away from the bowel wall to allow for current concentration to a relatively small area and limit thermal damage to the submucosa. As the area of contact between the metal cups of the forceps is relatively large, the current in the tissue can be sizeable, resulting in a significant thermal effect. A deep thermal effect can cause delayed wall necrosis and perforation, particularly in areas where the digestive wall is thin, such as the cecum and ascending colon.

\subsubsection{Risk of explosion during colon polypectomy}

Several case reports have described explosions occurring at the time of electrosurgery in the colon. The risk of explosion exists when combustible gas concentrations are elevated in the colon (methane $>5 \%$, hydrogen $>4 \%$, and oxygen $>5 \%$ ) [13]. These conditions may be present in poorly prepped or unprepped colons, or when mannitol-based bowel preparations are used [14]. With the advent of polyethylene glycol or sodium phosphate-based preparations for bowel cleansing, this concern has markedly decreased. Using carbon dioxide for gut distension during endoscopy can also minimize the explosion risk.

\subsection{Sphincterotomy}

\subsubsection{Sphincterotomes}

Sphincterotomes consisting of monofilament or braided cutting wires are commercially available. Monofilament sphincterotomes may provide a more clear-cut incision with less risk of heat injury at the sphincterotomy edges and the ampullary area but, to our knowledge, a prospective randomized trial comparing the two devices has yet to be performed. One of the available sphincterotomes (CleverCut, Olympus Inc., Tokyo, Japan) has the proximal part of the exposed wire covered with insulation material to decrease contact between the wire and the endoscope or surrounding tissues. Sphincterotomes may have one, two or three independent lumens to accept the cutting wire, a guide wire, and contrast medium. Single-lumen sphincterotomes are rarely used as the guide wire should be removed during sphincterotomy (electric short circuits may develop between the cutting wire and the guide wire if guide wire insulation is defective, inducing lesions at the distal end of the guide wire) [15]. A double-lumen sphincterotome equipped with an angled Tuohy-Borst valve (DASH, Wilson Cook Inc., Winston-Salem, North Carolina, USA) allows injection of contrast medium when a guide wire has been inserted into the sphincterotome because the valve prevents spillage of contrast medium during injection. Rotatable sphincterotomes (e.g. Autotome, Boston Scientific Inc., Natick, Massachusetts, USA) may be useful, particularly in difficult situations such as Billroth II anatomy.

\subsubsection{Current application}

Factors associated with a higher effectiveness for cut initiation and propagation during biliary sphincterotomy in an experimental setting include a smaller diameter of the electrode wire, a shorter length of wire in contact with the tissue, a higher force applied with the wire onto tissue, and higher power settings [16]. Practical issues related to sphincterotomy are as follows. 
- If no effect is observed within one or two seconds after current application, it may be useful to reduce the length of wire in contact with the surrounding tissue.

- Uncontrolled rapid cutting ("zipper effect"), a potentially dangerous event, may develop as the length of wire in contact with the tissue decreases (e.g. once tissue has been cut) or if the force applied with the sphincterotome is high.

The type of current used for sphincterotomy (pure cut vs. blended) does not influence the incidence or severity of pancreatitis, the most frequent complication of sphincterotomy, as shown in a meta-analysis of randomized trials [17]. One of the trials in the meta-analysis, in which the Endocut mode was used to provide blended current, failed to show any significant difference in terms of postsphincterotomy pancreatitis with Endocut vs. pure cut current [18]. However, pure cut current was associated with a higher incidence of bleeding. Blended current is recommended for biliary sphincterotomy, particularly in patients at high risk of bleeding [19]. Current modes that provide alternating cutting and coagulation phases (e.g. Endocut, Pulsecut) are increasingly being used, in part due to the impression that they provide better control of the cut. The Endocut I mode has been specifically designed for sphincterotomy but has not been validated in controlled trials.

As heating related to coagulation is thought to favor the development of local edema that might obstruct pancreatic outflow, the combination of current waveforms has been explored as a potential means to reduce postsphincterotomy pancreatitis (e.g. pure cut current for sphincterotomy initiation followed by blended current after having cut 3-5 mm of tissue). However, comparative studies of combined current waveforms vs. pure cut or blended current used throughout sphincterotomy have found no difference in the outcome of postsphincterotomy pancreatitis $[20,21]$.

\subsection{Hemostasis}

All ESUs offer coagulation modes that can be applied immediately during a cutting procedure using a nonspecific device (e.g. applying coagulation current at the bleeding edge of a biliary sphincterotomy using the sphincterotome wire) or to treat a spontaneously bleeding lesion with a specific device (e.g. bipolar coagulation probe).

A range of devices are available for either monopolar or bipolar coagulation. These devices include APC probes, coagulating forceps, and bipolar probes. These latter devices require contact with tissue and allow for immediate hemostasis by compressing the bleeding vessel (tamponade). APC is particularly useful in treating large surface areas; it should be used with caution in the vicinity of hemostatic endoclips, as the latter are current conductors.

APC is a noncontact, monopolar, electrocoagulation technique that works by applying high frequency current to the tissues through ionized argon (argon is an inert gas that requires to be ionized to conduct current). As the current exiting the tip of the APC probe follows the path of least resistance, tissues that are already coagulated (and, hence, of increased electrical resistance) receive less current than surrounding tissues. The high frequency current is applied while attempting to avoid direct contact between the probe and the bowel wall in order to avoid gas injection into tissue (pneumatosis). Compared with contact methods of coagulation, APC provides a more homogeneous tissue effect and can treat large surface areas more rapidly. Also, it may harbor a lower perforation risk because the penetration depth is limited to 1-3 mm [22]. However, APC lacks the mechanical (tamponade) hemostatic effect of contact techniques. APC is mainly used for:

- coagulating superficial vascular lesions (e.g. sporadic angiodysplasias, gastric antral vascular ectasia, radiation proctitis);

- palliative ablation of tumors, in particular tissue ingrowth/ overgrowth of esophageal stents;

- ablation of residual polyp tissue after piecemeal resection of large sessile polyps [23];

- transection of metal stents at high power settings (e.g. migrated biliary stents impinging onto the contralateral duodenal wall, causing obstruction and ulcers) [24].

Practical recommendations regarding APC use include adequate bowel cleansing (for colonic applications), avoidance of APC in the vicinity of metallic devices (e.g. clips), and intermittent suctioning of the argon gas to minimize gut distension.

\subsection{Endoscopic mucosal resection and submucosal dissection}

Endocut and Pulsecut are commonly used settings for endoscopic mucosal resection (EMR) and ESD, although some endoscopists use pure cut waveforms. Compared with EMR, ESD is technically more challenging and time consuming but enables en bloc resection in most cases. The instruments (knives) used for dissection and the type of current waveforms and power settings selected are influenced by various parameters, such as lesion location, tissue characteristics, and operator preferences. As the technique of ESD is not yet standardized, endoscopists mainly rely on personal or reported experience thus far.

\section{Monopolar vs. bipolar accessories}

\section{$\nabla$}

All ESUs used in digestive endoscopy provide monopolar and bipolar modes; few provide bipolar cutting current. This is due to the limited availability of devices adapted for bipolar use apart from coagulation probes. Prototype bipolar polypectomy snares [7], hot biopsy forceps [25], sphincterotomes, and needle knife for ESD $[26,27]$ have been developed to profit from their theoretical advantages over monopolar devices (i.e. less power to achieve similar effects and more superficial delivery of energy with decreased risk of perforation) $[25,28]$. However, these bipolar devices are not widely available, in part due to their more complex design and fabrication costs relative to their monopolar counterparts.

Bipolar mode is currently used almost exclusively with catheter probes designed for hemostasis of nonvariceal bleeding lesions. This type of probe provides treatment outcomes similar to other effective modalities [29]. Bipolar probes usually permit irrigation, which is particularly useful if an endoscope with a single working channel is used.

\section{Conclusion}

$\checkmark$

Digestive endoscopy has been developing as a therapeutic tool for more than 30 years, with electrosurgery being an essential part of this development. In electrosurgery, high frequency current is used to produce heat in tissue. Various parameters, including current waveform, power setting, type of active electrode, and duration of current delivery allow control of tissue heating and the resulting outcome of cut and/or coagulation of tissue. 
The safe and effective performance of electrosurgery during endoscopy requires an understanding of these parameters.

\section{Acknowledgment}

The authors thank P. Dumonceau for assistance with generating the figures.

Competing interests: Representatives from the following companies participated in an advisory capacity: J. Raiser (Erbe Elektromedizin, Tübingen, Germany), I. Mesecke-von Rheinbaben (Olympus Europa, Hamburg, Germany), T. Carter (Boston Scientific, Hemel Hempstead, UK), and C. Tockweller (Gebruder Martin GmbH, Tuttlingen, Germany).

\section{References}

1 Cushing $H$. Electro-surgery as an aid to the removal of intracranial tumors. Surg Gynecol Obstet 1928; 47: 751 - 784

2 Wright VC. Contemporary electrosurgery: physics for physicians. J Fam Pract 1994; 39: 119-122

3 Curtiss LE. High frequency currents in endoscopy: a review of principles and precautions. Gastrointest Endosc 1973; 20: 9-12

4 Morris ML, Tucker RD, Baron TH, Song LMWK. Electrosurgery in gastrointestinal endoscopy: principles to practice. Am J Gastroenterol 2009; 104: $1563-1574$

5 American Society of Anesthesiologists Task Force on Perioperative Management of Patients with Cardiac Rhythm Management Devices. Practice advisory for the perioperative management of patients with cardiac rhythm management devices: pacemakers and implantable cardioverter-defibrillators. Anesthesiology 2005; 103: 186-198

6 Parra-Blanco A, Kaminaga N, Kojima T et al. Colonoscopic polypectomy with cutting current: is it safe? Gastrointest Endosc 2000; 51: 676681

7 Tucker RD, Platz CE, Sievert CE et al. In vivo evaluation of monopolar versus bipolar electrosurgical polypectomy snares. Am J Gastroenterol 1990; 85: $1386-1390$

$8 \mathrm{Kim}$ HS, Kim TI, Kim WH et al. Risk factors for immediate postpolypectomy bleeding of the colon: a multicenter study. Am J Gastroenterol 2006; 101: $1333-1341$

9 Van Gossum A, Cozzoli A, Adler M et al. Colonoscopic snare polypectomy: analysis of 1485 resections comparing two types of current. Gastrointest Endosc 1992; 38: 472 - 475

10 Fry LC, Lazenby AJ, Mikolaenko I et al. Diagnostic quality of polyps resected by snare polypectomy: does the type of electrosurgical current used matter? Am J Gastroenterol 2006; 101: 2123-2127

11 Singh N, Harrison M, Rex DK. A survey of colonoscopic polypectomy practices among clinical gastroenterologists. Gastrointest Endosc 2004; 60: 414-418

12 Vanagunas A, Jacob P, Vakil N. Adequacy of "hot biopsy" for the treatment of diminutive polyps: a prospective randomized trial. Am J Gastroenterol 1989; 84: 383-385
13 Monahan DW, Peluso FE, Goldner F. Combustible colonic gas levels during flexible sigmoidoscopy and colonoscopy. Gastrointest Endosc 1992; 38: 40-43

14 Avgerinos A, Kalantzis N, Rekoumis $G$ et al. Bowel preparation and the risk of explosion during colonoscopic polypectomy. Gut 1984; 25 : $361-364$

15 Johlin FC, Tucker RD, Ferguson S. The effect of guidewires during electrosurgical sphincterotomy. Gastrointest Endosc 1992; 38: 536-540

16 Ratani RS, Mills TN, Ainley CC, Swain CP. Electrophysical factors influencing endoscopic sphincterotomy. Gastrointest Endosc 1999; 49: 43-52

17 Verma D, Kapadia A, Adler DG. Pure versus mixed electrosurgical current for endoscopic biliary sphincterotomy: a meta-analysis of adverse outcomes. Gastrointest Endosc 2007; 66: 283 - 290

18 Norton ID, Petersen BT, Bosco J et al. A randomized trial of endoscopic biliary sphincterotomy using pure-cut versus combined cut and coagulation waveforms. Clin Gastroenterol Hepatol 2005; 3: 1029-1033

19 Dumonceau JM, Andriulli A, Deviere J et al. European Society of Gastrointestinal Endoscopy (ESGE) Guideline: prophylaxis of post-ERCP pancreatitis. Endoscopy 2010; 42: 503-515

20 Gorelick A, Cannon M, Barnett J et al. First cut, then blend: an electrocautery technique affecting bleeding at sphincterotomy. Endoscopy 2001; 33: $976-980$

21 Stefanidis G, Karamanolis G, Viazis $N$ et al. A comparative study of postendoscopic sphincterotomy complications with various types of electrosurgical current in patients with choledocholithiasis. Gastrointest Endosc 2003; 57: $192-197$

22 Farin G, Grund KE. Technology of argon plasma coagulation with particular regard to endoscopic applications. Endosc Surg Allied Technol 1994; 2: $71-77$

23 BrookerJC, Saunders BP, Shah SG et al. Treatment with argon plasma coagulation reduces recurrence after piecemeal resection of large sessile colonic polyps: a randomized trial and recommendations. Gastrointest Endosc 2002; 55: 371 - 375

24 Manner H, Enderle MD, Pech O et al. Second-generation argon plasma coagulation: two-center experience with 600 patients. J Gastroenterol Hepatol 2008; 23: 872-878

25 Savides TJ, See JA, Jensen DM et al. Randomized controlled study of injury in the canine right colon from simultaneous biopsy and coagulation with different hot biopsy forceps. Gastrointest Endosc 1995; 42: $573-578$

26 Saito Y, Uraoka T, Matsuda T et al. Endoscopic treatment of large superficial colorectal tumors: a case series of 200 endoscopic submucosal dissections (with video). Gastrointest Endosc 2007; 66: 966-973

27 Siegel JH, Veerappan A, Tucker R. Bipolar versus monopolar sphincterotomy: a prospective trial. Am J Gastroenterol 1994; 89: 1827-1830

28 Tucker RD, Sievert CE, Platz CE et al. Bipolar electrosurgical sphincterotomy. Gastrointest Endosc 1992; 38: 113-117

29 Laine L, McQuaid KR. Endoscopic therapy for bleeding ulcers: an evidence-based approach based on meta-analyses of randomized controlled trials. Clin Gastroenterol Hepatol 2009; 7: 33-47; quiz 31 - 32

Appendices 1 and 2 are available online: www.thieme-connect.com/media/endoscopy/supmat/en146.pdf 